\title{
Recent Advances in Blood-Brain Barrier Disruption as a CNS Delivery Strategy
}

\author{
Marc-André Bellavance, ${ }^{1}$ Marie Blanchette, ${ }^{2}$ and David Fortin ${ }^{3,4,5}$
}

Received 6 November 2007; accepted 13 February 2008; published online 18 March 2008

\begin{abstract}
The blood-brain barrier (BBB) is a complex functional barrier composed of endothelial cells, pericytes, astrocytic endfeets and neuronal cells. This highly organized complex express a selective permeability for molecules that bear, amongst other parameters, adequate molecular weight and sufficient liposolubility. Unfortunately, very few therapeutic agents currently available do cross the BBB and enters the CNS. As the BBB limitation is more and more acknowledged, many innovative surgical and pharmacological strategies have been developed to circumvent it. This review focuses particularly on the osmotic opening of the BBB, a well-documented approach intended to breach the BBB. Since its inception by Rapoport in 1972, pre-clinical studies have provided important information on the extent of BBB permeation. Thanks to Neuwelt and colleagues, the osmotic opening of the BBB made its way to the clinic. However, many questions remain as to the detailed physiology of the procedure, and its best application to the clinic. Using different tools, amongst which MRI as a real-time in vivo characterization of the BBB permeability and CNS delivery, we attempt to better define the osmotic BBB permeabilization physiology. These ongoing studies are described, and data related to spatial and temporal distribution of a molecule after osmotic BBB breaching, as well as the window of BBB permeabilization, are discussed. We also summarize recent clinical series highlighting promising results in the application of this procedure to maximize delivery of chemotherapy in the treatment of brain tumor patients.
\end{abstract}

KEY WORDS: blood-brain barrier, blood-brain barrier disruption, brain tumors, CNS delivery; MRI.

\section{INTRODUCTION}

Systemic treatment of many central nervous system (CNS) diseases, such as brain tumors, is considerably impaired by limited delivery of therapeutics. Poor CNS access is mainly related to selective barriers that sequester the CNS from the circulatory system (1). In 1885, Paul Ehrlich was the first to demonstrate experimentally that the brain is a sanctuary site $(2,3)$. In contrast to peripheral organs, he observed a lack of CNS coloration after intravenous injection of vital dyes. Almost 30 years later, Ehrlich's student Edwin Goldmann noticed the opposite phenomenon following injection of these dyes in the cerebrospinal fluid (CSF; 4). The staining was then confined to the CNS and peripheral organs were exempted of any coloration, suggesting the

\footnotetext{
${ }^{1}$ Department of Pharmacology, Sherbrooke University, Sherbrooke, Québec, Canada.

${ }^{2}$ Department of Radiobiology and Nuclear Medicine, Sherbrooke University, Sherbrooke, Québec, Canada.

${ }^{3}$ Department of Surgery, division of Neurosurgery and Neurooncology, Sherbrooke University, Sherbrooke, Québec, Canada.

${ }^{4}$ CHUS, Sherbrooke University, 3001, 12e Ave Nord, Sherbrooke, Québec J1H 5N4, Canada.

${ }^{5}$ To whom correspondence should be addressed. (e-mail: david. fortin@usherbrooke.ca)
}

presence of a barrier between the CNS and the circulation. Research endeavour that followed these pioneer works ascribed this observation to the BBB. Located at the blood/ brain interface, this barrier represents the primary obstacle to CNS penetration for blood circulating therapeutics (5).

The BBB is a complex functional barrier collectively composed of endothelial cells, pericytes, astrocytic endfeets and neuronal cells (6-9). This highly organized complex, also known as the neurovascular unit, confers unique properties to the CNS endothelium accounting for the selective permeability of the BBB. Endothelium tight junctions, lack of fenestrae and low pinocytic/endosomal transport prevent the diffusion of hydrosoluble molecules across the BBB (10-12). Moreover, astrocytic endfeet configuration practically seals CNS vessels by covering nearly $99 \%$ of their external surface (1315). This peculiar organization of the BBB restrains passive transport (non-receptor or non-carrier mediated transport) to the CNS compartment from the blood.

To achieve CNS entry in the presence of an intact BBB, a molecule must weight less than $180 \mathrm{Da}$ and bears a sufficient liposolubility $(16,17)$. The latter is defined by an optimal oil/water partition coefficient value of 1.6 or by the formation of less than eight to ten hydrogen bonds with solvent water (18-20). These properties are the most decisive, but the degree of ionization, plasma protein binding, local cerebral blood flow and affinity for dedicated carriers can also influence the BBB permeabilization (21). Unfortunately, very 
few molecules meet these characteristics, and even for the ones who do, CNS entry is not assured $(22,23)$. In fact, highly active efflux pumps, such as P-glycoprotein (P-gp), offers an additional layer of protection to the CNS by expelling harmful molecules back in the blood $(24,25)$. As a consequence, $98 \%$ of small molecules and the totality of large molecular weight drugs are excluded from the CNS compartment (19). The BBB thus regulates the fragile brain homeostasis by strictly controlling the composition of the CSF and extracellular fluid compartment via entrance and withdrawal of specific compounds at the blood-brain interface.

\section{The Altered BBB in the Context of Brain Neoplasia}

Although the integrity of the barrier is often compromised within a tumor, this alteration in permeability is variable and dependent on tumor type and size. Moreover, it is extremely heterogeneous in a given lesion. Although the $\mathrm{BBB}$ is frequently leaky in the center of malignant brain tumors, the well-vascularized actively proliferating edge of the tumor (the brain adjacent to tumor or BAT), has been shown to have variable and complex barrier integrity (26). Therefore, by steeply reducing the concentration of intravenously administered chemotherapeutic agent at the periphery of the tumor, this sink effect phenomenon is yet another mechanism that can contribute to chemotherapy failure in CNS neoplasm treatment.

Despite increasing recognition, this topic remains considerably underdeveloped in the field of neurosciences. More so, it is said that if a pharmaceutical company decided to develop a research program on the $\mathrm{BBB}$, there would be few BBB-trained scientists to hire, because less than $1 \%$ of U.S. academic neuroscience programs emphasize BBB transport biology (27).

\section{Bypassing the BBB}

In spite of the limitations imposed by the BBB, transvascular delivery still represents, in our view, the ideal global dissemination channel to the CNS. The extensiveness of the cerebral vascular network insures a quick and global way to reach the CNS, if the mechanisms collectively called BBB can be bypassed (28-30).

Many innovative strategies have been developed to circumvent the BBB in order to increase drug delivery to the CNS. These approaches can be broadly classified under two headings: surgical and pharmacological. Surgical approaches typically involve a more invasive paradigm. Trans-nasal delivery routes, and transcranial delivery, either via intracerebral implants or via convection enhanced delivery (CED), are classified under this heading. Osmotic BBB disruption, which involves placement of an intra-arterial catheter and general anesthesia is also considered a surgical approach.

Pharmacological oriented strategies involve drug modification, pro-drug synthesis, and the use of pharmacological agents to breach the $\mathrm{BBB}$, such as bradykinin agonists, like RMP-7. Other investigators are exploiting the presence of dedicated carrier or receptor-mediated transport systems, also classified as pharmacological-oriented strategy.

We elected to work with osmotic blood-brain barrier disruption (BBBD), as this approach is already in clinical use and provide a global hemispheric delivery paradigm (16). However, by no means do we imply that it is the sole or the best approach to gain access to the CNS. As a matter of fact, our lab is also studying and developing pharmacological approaches to breach the BBB.

\section{OSMOTIC BLOOD BRAIN BARRIER DISRUPTION}

In 1972, Rapoport et al. reported CNS tissue staining by Evans Blue consequently to intra-arterial infusion of hypertonic arabinose (26). Since Evans Blue, which binds to albumin, is not expected to permeate through the intact $\mathrm{BBB}$, this observation suggested a BBB alteration by hypertonic arabinose. The authors proposed that hypertonic solutions increased BBB permeability by inducing the shrinkage of cerebrovascular endothelial cells, thus producing a disruption of inter-endothelial tight junctions (31,32). The concept of osmotic BBBD was then introduced. Brightman and coworkers later supported this hypothesis, as they

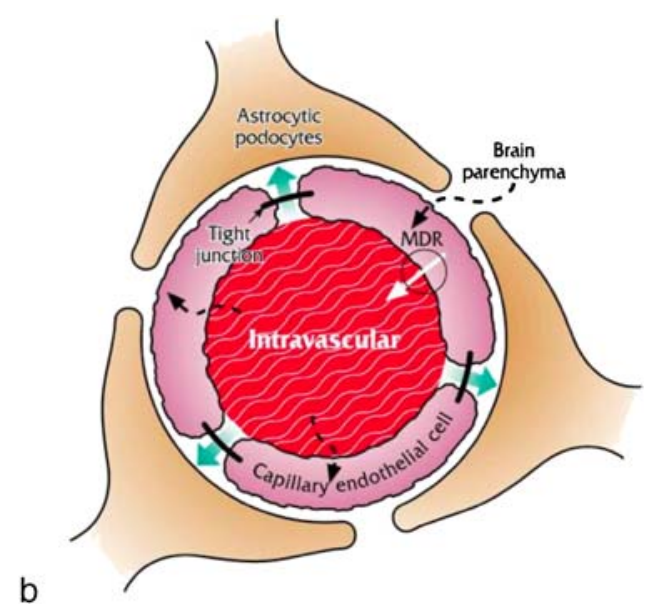

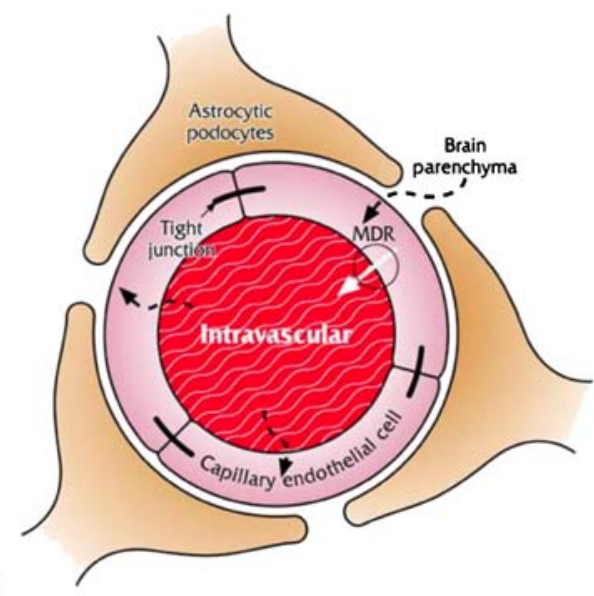

a

$\mathrm{b}$

Fig. 1. Graphical sketch illustrating the hypothesis concerning the osmotic BBB modification. The tight junctions are shown a as devoid of any anatomic space between the endothelial cells. Moreover, multi-drug resistance (MDR) gene products, such as the P-gp efflux pump, are also illustrated as they are integral to the mechanism of the barrier. The osmotic BBBD procedure induces a retraction in the cell membrane, and a physical opening $\mathbf{b}$ accompanied by a modification of the $\mathrm{Ca}^{2+}$ metabolism in the cell 
successfully visualized opened endothelial tight junctions with electron miscroscopy after intra-carotid infusion of mannitol in several species (32). In 1984, Dorovini-zis et al. also observed opened inter-endothelial tight junctions when endothelial cell cultures were exposed to a hypertonic solution (33). Although the precise mechanism of osmotic BBBD is still debated, such works support the concept of tight junctions physical disruption by shrinkage of CNS endothelial cells (Fig. 1).

Besides arabinose, hyperosmolar solutions of lactamide, saline, urea, radiographic contrast agents and mannitol can be used to transiently breach the BBB (21). The latter is commonly used for BBBD in both animals and humans since its administration has been approved many years before the implementation of this technique. Since its initial description, the procedure has been used in preclinical and even clinical studies (34-37).

In this review, we intend to present information in a translational continuum, reflecting the knowledge acquired on the procedure from the pre-clinical field to the clinical application of BBBD.

\section{Preclinical Data}

Different BBBD animal models have been described in the literature, but the rat model is the most reported on, because of its ease of use (38). In these animals, the procedure is standardized, and has been described in detail in numerous studies (39-41). Briefly, after general anesthesia and endotracheal intubation, the carotid complex is exposed, and the external carotid artery is isolated. After incision and retrograde cannulation of the artery with a PE-50 tubing, the tip of the catheter is positioned near the carotid bifurcation. The hypertonic solution of mannitol is then infused in a retrograde fashion in the internal carotid artery, followed by the administration of the therapeutic agent (42).

The work of different investigators suggested that the anesthetic agents must be chosen carefully because their hemodynamic effects can impact on the quality of the BBB opening $(43,44)$. We initiated our pre-clinical studies with ketamine/xylazine, and had to design a modification to the model to circumvent the negative impact of ketamine on the cerebral blood flow that ultimately affected the extent of BBBD (44). This was accomplished by simply isolating the perfused hemisphere from the systemic circulation by applying a temporary vascular clip to the common carotid artery, prior to the infusion of mannitol (Fig. 2). This prevented backflow of mannitol in the cardiac chamber, and increased the extent and reliability of the procedure (42).

Ultimately, however, propofol proved to be the anesthetic agent of choice as it improved both the efficiency and consistency of the disruption procedure in animals (43). As propofol is infused continuously (iv) and has a very short halflife, it can be easily titrated, insuring a better control over vital signs than with ketamine. More so, propofol is an antiseizure medication and a cerebro-protectant, thus conferring to this anesthetic the ideal profile for the BBBD procedure.

However, as the use of propofol translates in a better delivery, the drawback we observed was an increase in neurotoxicity with some co-administered chemotherapeutic

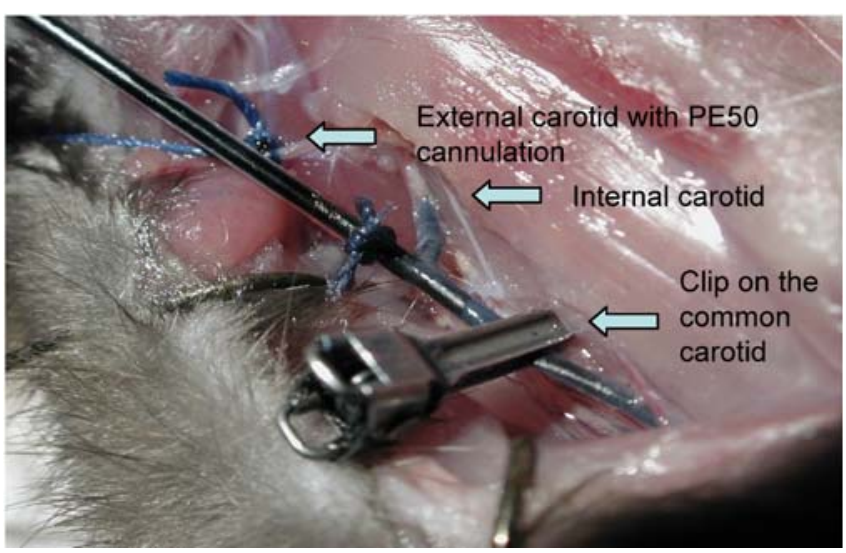

Fig. 2. Surgical setup for BBBD. The carotid complex was exposed, and the external carotid artery was ligated and incised. A PE50 tubing was inserted in a retrograde fashion, and was positioned just above the bifurcation. The tubing was stabilized with a suture. Notice the clip on the common carotid artery, used to prevent backflow during mannitol infusion

agents otherwise known to be safe when paired to other anesthetics (45).

\section{Pre-clinical Quantitative Studies}

\section{Albumin}

Several factors can negatively impact the effectiveness of the BBBD; hemodynamic variables, anesthetic agent and rate of infusion of hypertonic solutions are but a few examples $(43,46)$. As a consequence, even repeated procedures in the same individual or animal can lead to highly variable BBB disruptions. It is thus paramount to monitor the extent of $\mathrm{BBB}$ permeation for each procedure, if we wish to study the actual influence in the intensity of delivery against different outcome.

This can be accomplished in the clinic with the use of a contrast-enhanced CT scan performed shortly after BBBD. The magnitude of BBBD is then evaluated in a semi-quantitative fashion, using visual scale (47). A similar principle has been described by Rapoport in pre-clinical studies, where the contrast-enhanced CT scan is substituted by a macroscopic staining of the rat cerebrum using Evans blue, a staining compound that binds the albumin $(48,49)$. The staining at the surface of the brain is evaluated against an arbitrary staining scale, returning a qualitative evaluation in the intensity of delivery (16,20; Fig. 3). This approach is inherently subjective and the reliability of the generated results is therefore questionable. More so, the fact that the Evans blue so tightly binds albumin is questioned nowadays. This is obviously a critical issue on which the whole concept is based. Another weakness of this approach arises from the global superficial evaluation, in that only the cortical surface is surveyed; the deep structures are not visualized and no data is gain on the topographic extent of delivery.

To overcome these shortcomings, we adapted an approach based on albumin immunohistochemistry. This concept has been exploited in the past by Vorbrodt et al. to study the dynamics of BBBD (50). These authors used quantitative immunocytochemistry against albumin, fol- 


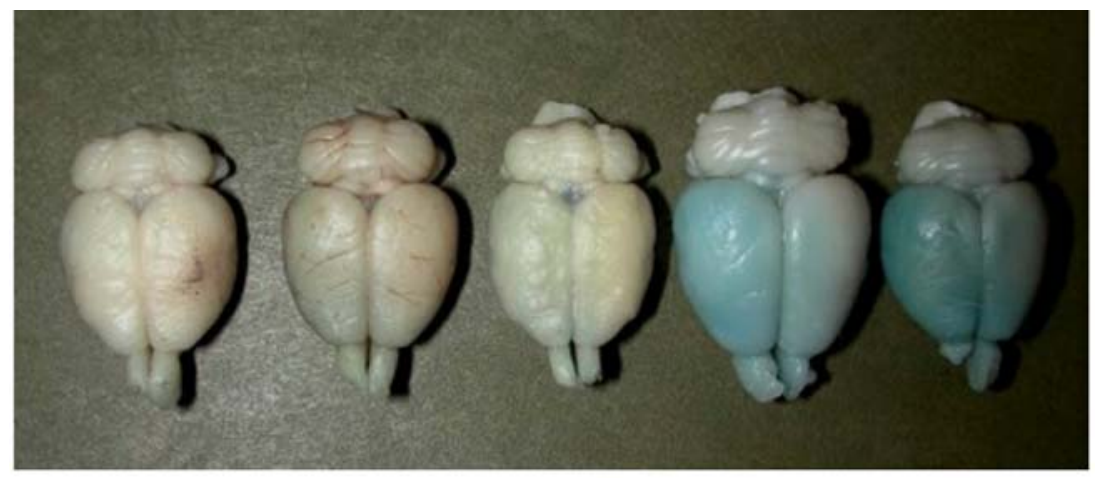

\section{$0.06 \mathrm{cc} / \mathrm{sec} \quad 0.08 \mathrm{cc} / \mathrm{sec} . \quad 0.10 \mathrm{cc} / \mathrm{sec} \quad 0.12 \mathrm{cc} / \mathrm{sec} \quad 0.15 \mathrm{cc} / \mathrm{sec}$}

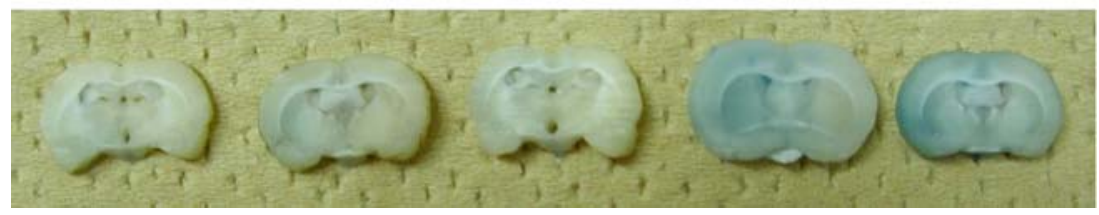

Fig. 3. Whole brain and corresponding coronal slices of samples extracted from one representative animal of each group exposed to different rates of mannitol infusion as assessed by Evans blue staining. A sequential increment in blue discoloration is obvious, peaking at $0.15 \mathrm{cc} / \mathrm{s}$

lowed by exposition to protein A-gold. The numerical value of their score was based on the square micrometer density of gold particles, and was used to detail the dynamics in the BBB permeabilization across different compartments. Thus the image analysis was performed on electron micrographs and did not allow a global estimation of delivery.

We designed a simple approach that allows the constitution of a precise data translating numerically the extent of delivery.

Briefly, we conducted osmotic BBBD with mannitol in adult male Fisher rats, with infusion rates ranging from 0.01 to $0.15 \mathrm{cc} / \mathrm{s}$. Brains were subsequently harvested and brain slices were processed for albumin immunochemistry, using a rabbit polyclonal antibody against albumin in a dilution of $1 / 100$. Biotinylated species-specific secondary antibody was applied (1/100) followed by an avidin-biotin amplification and peroxidase development. Afterwards, brain slices were digitized and ratios of stained pixels on total brain pixels were calculated. Percentages of delivery were obtained from these ratios (Fig. 4).

This data allows the production of a conservative estimate on the extent of delivery, as albumin is a large protein. It is expressed as a percentage of the treated hemisphere on a given coronal slice, and can also be used as a composite score translating global delivery, by simply summating the scores obtained on multiple contiguous slices. As the brain samples are cut in a standardized fashion using a brain matrix, the number of slices is always consistent, thus ensuring that the composite score is reproducible. Its objectiveness should help investigators to evaluate the impact of various surrogates on the CNS delivery and thus contribute to a better comprehension and characterization of the BBBD process. However, it has a significant drawback; sacrifice of the test
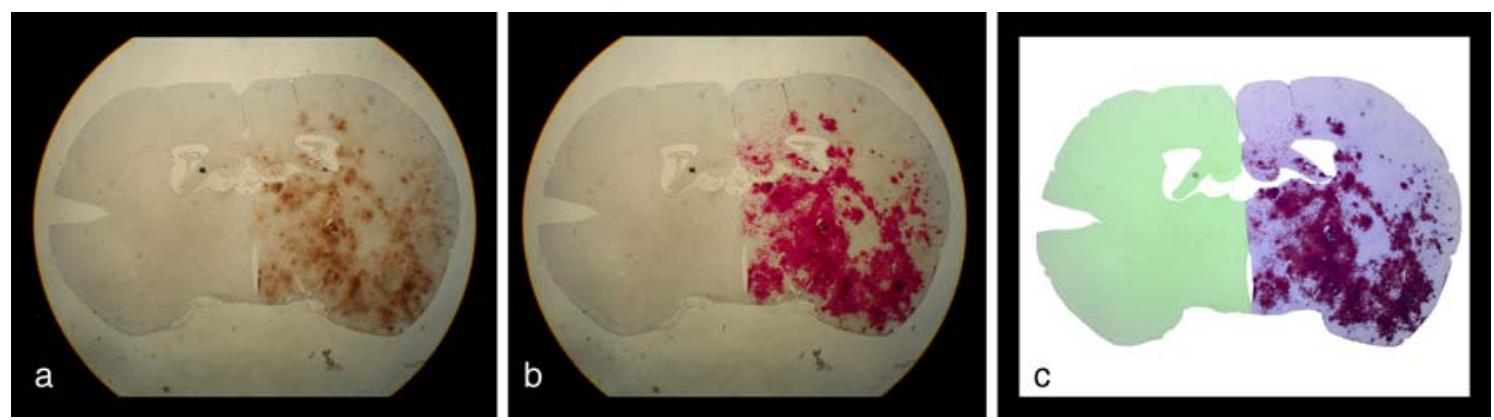

Fig. 4. Description of the steps involved in the calculation of the intensity of delivery ratio used in this study. a The albumin immunocytochemistry source image, displaying discolored areas in the treated hemisphere. b The image has been analyzed to identify pixels above a fixed threshold corresponding to the immunocytochemistry staining. These pixels are retained as the red overlay. c After having defined the pixel area of each hemisphere (green overlay is left hemisphere, whereas blue overlay is right hemisphere), the red overlay has been added to the image for final analysis. Results are expressed as number of stained pixels (red overlay) as a fraction (\%) of the treated (right) hemisphere (blue overlay). Results might also have been presented as the fraction of stained pixels (red overlay) over the entire slice area (green + blue overlays) 

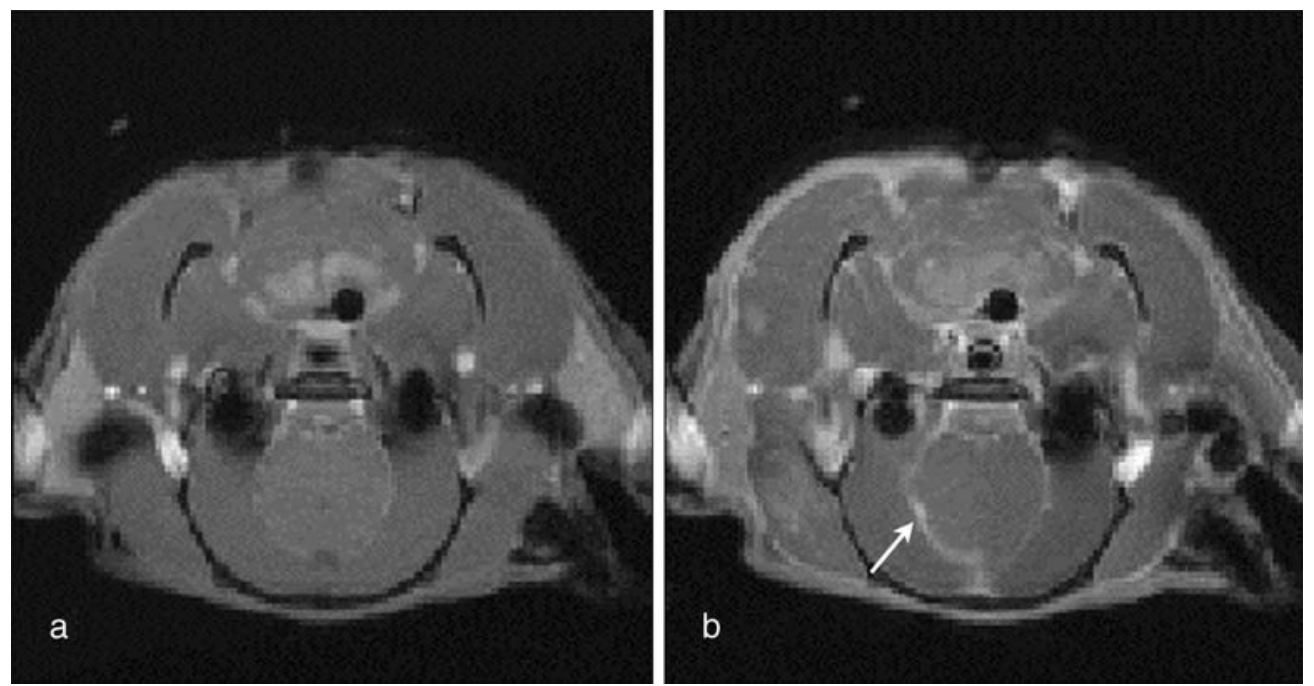

Fig. 5. An axial $\mathrm{T} 1$ enhanced cut, at the posterior aspect of the brain of a Wistar rat, prior (a) and after (b) BBBD. The arrow depicts the cleft area presenting the most intense increase in Gd-DTPA. It is from this area that a diffusion wave is observed

animals is required, thus prohibiting any kind of dynamic study over time. Hence, we are currently working on a different investigational approach allowing real time in vivo quantification of the BBB permeability and CNS delivery with MRI technology.

\section{MRI Quantification of BBB Permeability}

To palliate this lack of dynamic studies in the BBBD process, we have designed a methodology allowing osmotic $\mathrm{BBBD}$ to be conducted directly in the animal MRI gantry, thus permitting the acquisition of images dynamically before, during and after the procedure. This setup allows us to study the distribution of contrast agents of different molecular weight, to estimate brain exposure against several surrogates following the osmotic BBBD, and to estimate the window of barrier opening.

To evaluate the distribution of Gd-DTPA, a low molecular weight contrast agent $(0.9 \mathrm{KDa})$ within the brain of animals, MRI signal intensity was analyzed in several regions of interest (ROI) scattered across different areas of the brain over time. Initial results highlight the heterogeneity in the CNS penetration, and the subsequent diffusion of contrast agent from an area representing the cleft between the brainstem and the cerebral hemisphere (Fig. 5). This area was identified as consistently presenting the largest exposure to Gd-DTPA after BBBD. The

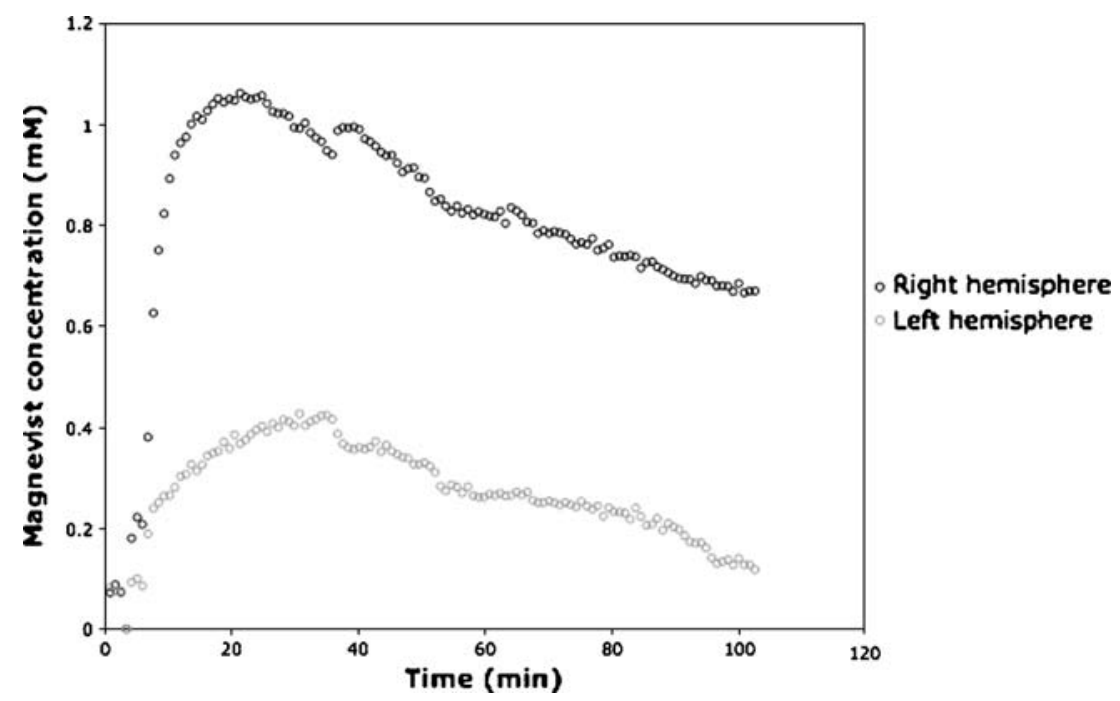

Fig. 6. Graphic depicting the Magnevist (Gd-DTPA) concentration over time in the disrupted hemisphere (right) and the non disrupted hemisphere (left) in T1 MRI sequence acquisition. Notice the pronounced signal drop at time $3 \mathrm{~min}$, immediately after BBBD, translating the increase in water content caused by the BBBD 
dynamics in the exposure of the brain to Gd-DTPA over time highlighted two different mechanisms by which the procedure increases $\mathrm{BBB}$ permeability: (1) a direct permeabilization of the BBB, (2) and a diffusion of the contrast agent within the brain from the area described above (Fig. 5).

The first phenomenon is a diffuse process of short duration observed in the whole treated hemisphere, and accounts for the initial T1 MRI signal drop, as water content is increased in the extracellular space (Fig. 6). The second mechanism involves a diffusion process in the interstitial compartment, and is directly affected by molecular size of the compound under study. This mechanism results in a delayed and broader distribution of the signal that eventually reach the contralateral hemisphere, producing a maximal exposure to the studied molecule much later. As an example, this delay is $66 \mathrm{~min}$ for Gd-DTPA. Interestingly, when studying molecules of a higher molecular weight, the distribution volume is more limited, as the large size of the molecule prevents free diffusion through CNS extracellular spaces. As an illustration of this, high molecular weight contrast agents such as Gadomer, a $17 \mathrm{kDa}$ synthetic dendrimer linked to paramag- netic Gadolinium molecules, hardly reach the opposite hemisphere (Blanchette et al., unpublished data).

To quantify brain exposure, initial brain tissue MRI signal intensity was recorded and subsequent signals measured during and after the BBBD were correlated to contrast agent concentrations by mathematical manipulations. Brain exposure can thus be assessed as a function of the amount of contrast agent in a given brain compartment (Fig. 7). As expected, overall brain parenchyma is more exposed to GdDTPA than Gadomer because of its lower molecular weight, allowing a wider volume of distribution. As a consequence, Gd-DTPA is also washed out more rapidly from the brain compartment than Gadomer. In fact, the latter appears to remain trapped in the brain for a longer period. These results suggest that both volume of distribution and persistence of a given molecule in the CNS is related to its molecular weight. Therefore, an equilibrium between these two parameters is the key to an optimal brain exposition.

To define the duration of $\mathrm{BBB}$ opening following the osmotic disruption, delayed administration of Gadomer $(17 \mathrm{kDa})$ is currently carried at different times after the osmotic
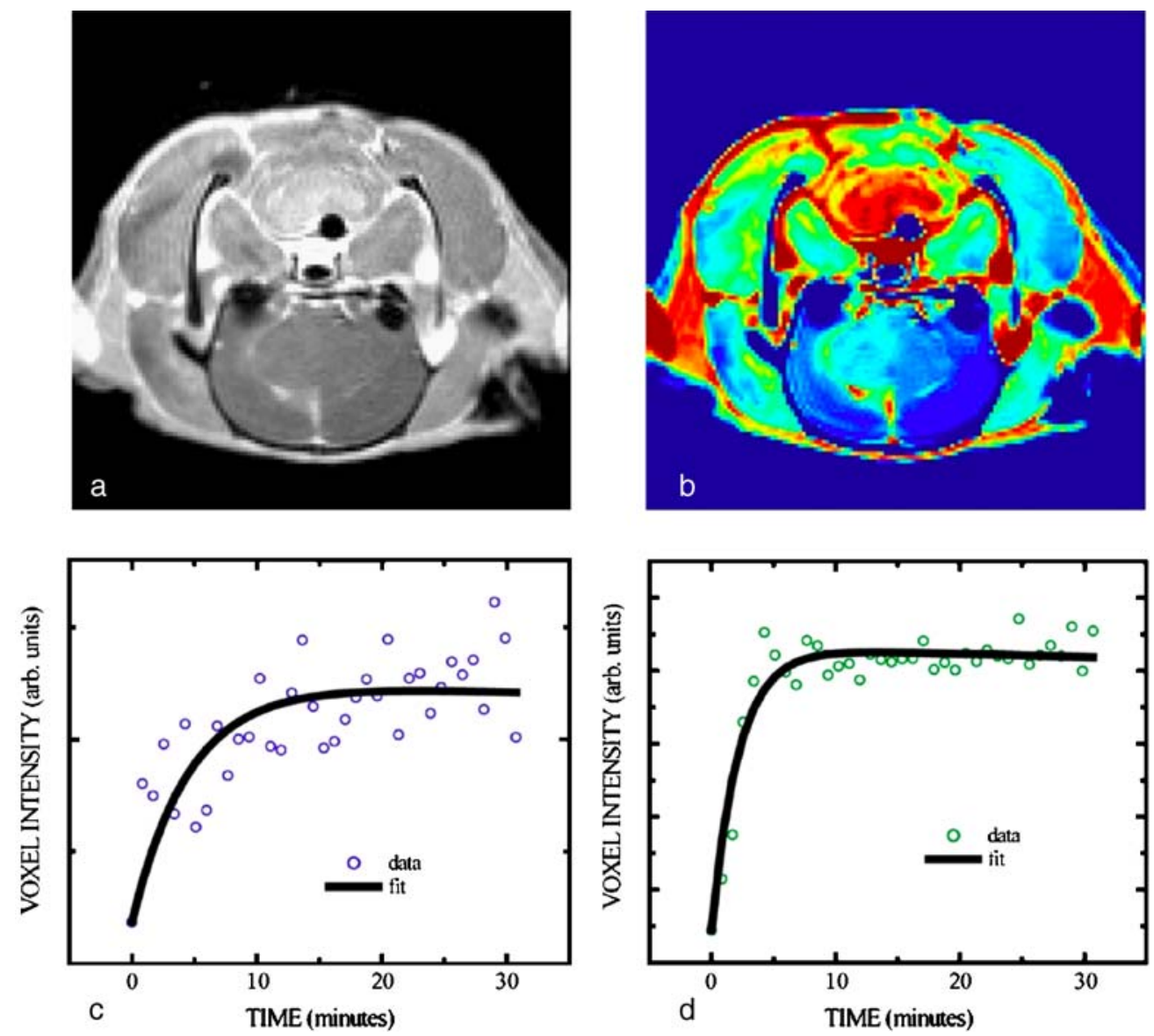

Fig. 7. An axial T1 Gd-DTPA enhanced MRI immediately after BBBD is analyzed using an exposition map translating the area under the curve (AUC). In this animal in which the intensity of the BBBD was moderate at best, the right hemisphere present a slight change in signal intensity (a). A map of the area under the curve (AUC) produced by the permeabilization procedure is presented in (b). Mathematical function extracted for a single pixel in the white matter of the right hemisphere. The increase in intensity is studied over time (c). Mathematical function extracted for a single pixel in the subependymal region of the right hemisphere (d). This area consistently depicts the most intense signal variation after osmotic BBBD 
BBBD procedure. Data obtained so far suggests that the BBB is still permeable to big molecules such as Gadomer up to $6 \mathrm{~h}$ after the osmotic BBBD (Fig. 8).

We are also in the process of studying the impact of BBBD on the activity of different efflux pumps, and on the brain metabolism with 18-FDG.

\section{CLINICAL STUDIES}

Thanks to the pioneering work of dedicated teams, the $\mathrm{BBBD}$ procedure has made its way to the clinic, and it has been extensively studied.

Neuwelt and his group conducted the first phase I clinical studies on osmotic opening of the blood-brain barrier, beginning in 1979 (16,36). Ever since, this group has been leading the clinical research effort on BBBD, joined by other institutions under the umbrella of the international BBBD consortium. A brief description of the clinical procedure will be detailed, followed by results obtained in clinical studies in the treatment of brain tumors.

\section{Description of the Clinical BBBD Procedure}

As general anesthesia is required, the osmotic opening of the $\mathrm{BBB}$ is accomplished in the operating room or in the angiography suite.

The human cerebral arterial system is organized in such a way that there basically are four major arteries responsible for the brain irrigation. The vascular anatomy can be variable from one individual to another, and thus the precise anatomy must be determined during the first treatment session by a formal cerebral angiography. The nature of the procedure, by produc- ing a vasogenic edema in the treated vascular distribution, do not allow for disruption of more than one vascular territory in a single treatment session. This implies that if a lesion covers more than one vascular distribution, or if there are multiple lesions, the treatment will be delivered in different distribution from cycle to cycle, to cover all relevant cerebrum territory.

Since hemodynamic factors impact on the degree of disruption, every attempt is made to keep heart rate and systemic pressure stable and above threshold values (established for each patient) during the general anesthesia $(36,51)$.

After general anesthesia, the technique involves the following steps:

1. Selective catheterization via percutaneous transfemoral puncture of left internal carotid artery, right internal carotid artery, left vertebral artery or right vertebral artery. The tip of the catheter is positioned at the $\mathrm{C} 2-\mathrm{C} 3$ vertebral level in the carotid, or at the C6-C7 vertebral level in the vertebral artery.

2. Determination of rate of infusion of mannitol by iodinated contrast injection and fluoroscopy, as the lowest infusion rate in which there is retrograde flow from the arterial catheter. The volume of mannitol infused will be the rate determined in $\mathrm{cc} / \mathrm{s} \times 30 \mathrm{~s}$. (usually between 4 to $10 \mathrm{cc} / \mathrm{s}$ in carotid circulation, and between 4 to $8 \mathrm{cc} / \mathrm{s}$ in vertebral circulation). The ultimate goal is to fill the entire vascular compartment in a given vessel distribution, without producing backflow of mannitol in the parent vessel.

3. The osmotic disruption is a physiologically stressful procedure. It can induce focal seizures in $5 \%$ of procedures (51). It can also trigger a vaso-vagal response

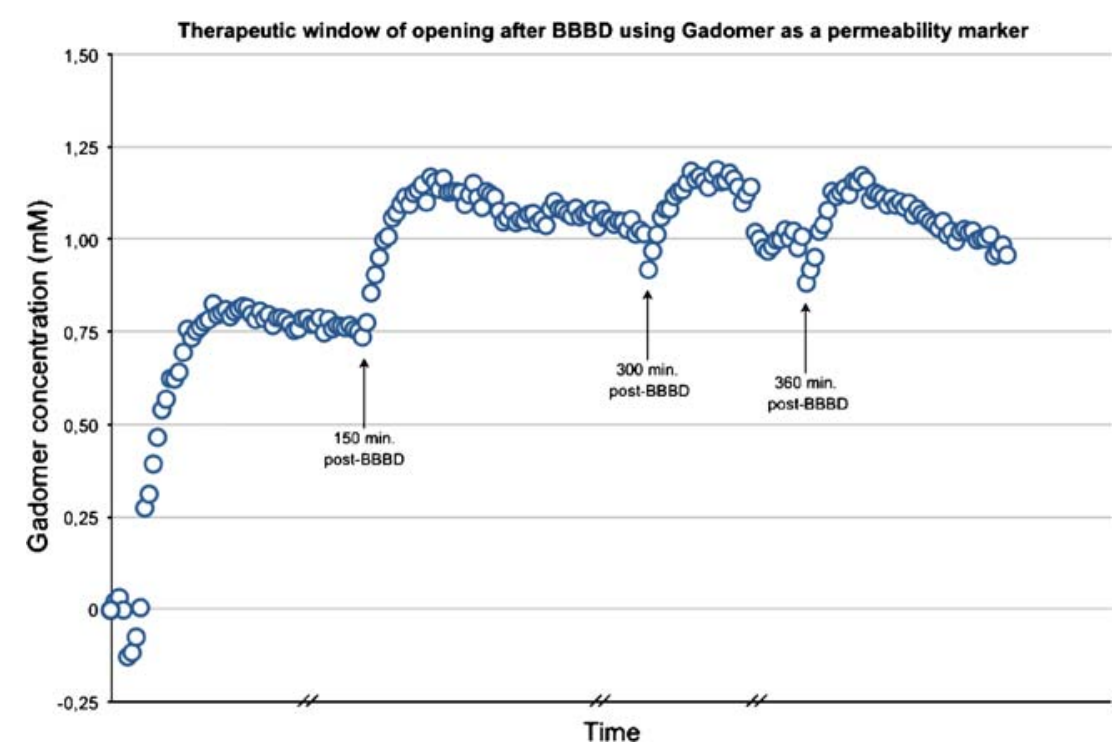

Fig. 8. Graphic depicting the Gadomer concentration over time in the disrupted hemisphere (right) in a selected region of interest of the frontal lobe. The first administration of Gadomer $(600 \mu \mathrm{l}$ of a $0.1429 \mathrm{mmol} / \mathrm{ml}$ solution at a rate of $500 \mu \mathrm{l} / \mathrm{min})$ was accomplished $3 \mathrm{~min}$ after BBBD. A significant increase in the concentration was observed. The infusion was repeated at 150, 300 and 360 min after BBBD. Notice small increments in concentration translating the residual permeability breach of the barrier that is still open at $360 \mathrm{~min}$ 
with bradycardia and hypotension. In order to prevent the occurrence of these adverse effects, different medications are administered just prior to the disruption to stabilize heart rate, pressure, and to produce neuro-protection.

4. Osmotic disruption of the blood brain barrier is accomplished by infusing mannitol $25 \%$ in the previously catheterized artery at the previously defined rate.

- During the infusion, interesting signs can be observed. The medial aspect of the forefront ipsilateral to the side of the infusion undergoes a whitish discoloration produced by the washout of blood from the ethmoidal branches, arteries known to connect the intracranial circulation to the extra cranial circulation. Bilateral pupillary dilatation can also be observed during the mannitol infusion. This is followed by a brief period of tachycardia and systemic hypertension.

5. Intra-arterial contrast is infused to confirm catheter position and rule out arterial injury post-disruption.

6. Infusion of the therapeutic molecule intra-arterially in the disrupted circulation. The concentration of the solution and the rate of infusion are critical factors when infusing intra-arterial solutions in avoiding neurotoxicity. The phenomenon of streaming defines an inhomogeneous distribution of the administered solution because of poor mixing at the infusion site. It is directly related to the Reynold number, a crucial parameter in fluid dynamics that predicts the transition from streamlined to turbulent flow (52). In the equation leading to the Reynold number, the density and viscosity of fluid, lumen diameter of the infused vessels and velocity of flow are all important determinants to control in order to avoid streaming (53).

7. Termination of procedure. The patient is taken to recovery room, where his neurological status and state of consciousness will regularly be evaluated until full recovery.

If the degree of BBBD opening needs to be assessed, a standard dose of IV non-ionic contrast bolus can be administered $5 \mathrm{~min}$ after the mannitol infusion. At the end of the procedure, before proceeding to the recovery room, the patient is taken to the CT scan suit.

\section{Contraindications to the BBBD Procedure}

The blood-brain barrier disruption procedure induces a transient rise in intra-cranial pressure (ICP) (baselines 3 to $9 \mathrm{~cm} \mathrm{H}_{2} \mathrm{O}$ to peaks of $16-23 \mathrm{~cm}$ at $30 \mathrm{~min}$ post disruption). This transient rise in ICP has been shown to correlate with a $1.5 \%$ increase in brain fluid content $(20,54)$. In pre-clinical studies, this transient increase in ICP was not associated with any clinical sequelae (20). It illustrates however the rationale behind the very first contraindication of osmotic BBBD: the presence of a significant mass effect. The definition of mass effect is somewhat arbitrary, but we uses standard clinical criteria to exclude patients deemed at risk.

Other contraindications to the procedure are also present, and include: evidence of spinal cord block from tumor mass, significant increased risk for general anesthesia and significant intra-arterial vascular pathologies.

\section{Adverse Effects}

In a seminal paper published by McAllister et al., the effect of the procedure combined to chemotherapy infusion (methotrexate) on neurocognitive function was evaluated in long term survivors from the procedure. Contrary to numerous established treatment modalities (e.g. cerebral radiotherapy), the BBBD procedure was not associated with any decline in formal neurocognitive assessment (55).

Adverse effects can nevertheless occur, and for ease of discussion, are stratified in two groups:

1- Catheter related complications

- Asymptomatic subintimal tear during catheterization (incidence 5\%)

- Significant groin hematoma post catheterization (incidence $0.5 \%$ )

- Parent vessel thrombosis, as experienced in two of our patients (incidence $0.5 \%$ ). This complication can produce long term neurological disability related to the vascular distribution involved. Fortunately, the occurrence of this complication did not translate in clinical repercussion in our patients.

\section{2- Disruption related complications}

- Seizures (incidence 5\%). Seizures are typically focal, and are immediately treated with IV thiopental and/ or IV Diazepam. This is typically a procedure related event.

- Temporary obtundation and/or increase in neurological symptoms (incidence $2.5 \%$ ). Complete recovery is the norm, and it typically last less than $48 \mathrm{~h}$. This adverse effect is associated with an excellent disruption.

- Brain herniation. Obviously, the most serious complication. One patient expired from this complication (incidence $0.3 \%$ ). It is the only mortality event related to the procedure in 300 patients that underwent a total of more than 3,000 procedures (51).

Doolittle et al. thus concluded that the procedure was safe, when performed in a standardized multicenter controlled setting (51).

\section{Clinical Results Obtained with the BBBD Procedure, in the Treatment of Brain Tumors}

Clinical results observed with the procedure will briefly be detailed below. However, before we initiate this discussion, a few key points first need to be addressed. Most clinical studies on BBBD have been conducted in patients with brain tumors, to increase delivery of chemotherapy. Some of these tumors, such as malignant astrocytomas, are notoriously chemoresistant, and as such, are not as likely to respond to chemotherapy as other type of cancers. Thus, two distinct factors are at play, difficult to segregate from each other in outcome studies: the delivery intensity, and chemosensitivity of the tumor. This limitation must be recognized when analyzing results from these studies. A negative study could be related to the choice of a poorly active therapeutic agent, even though this agent was adequately delivered to its target. 

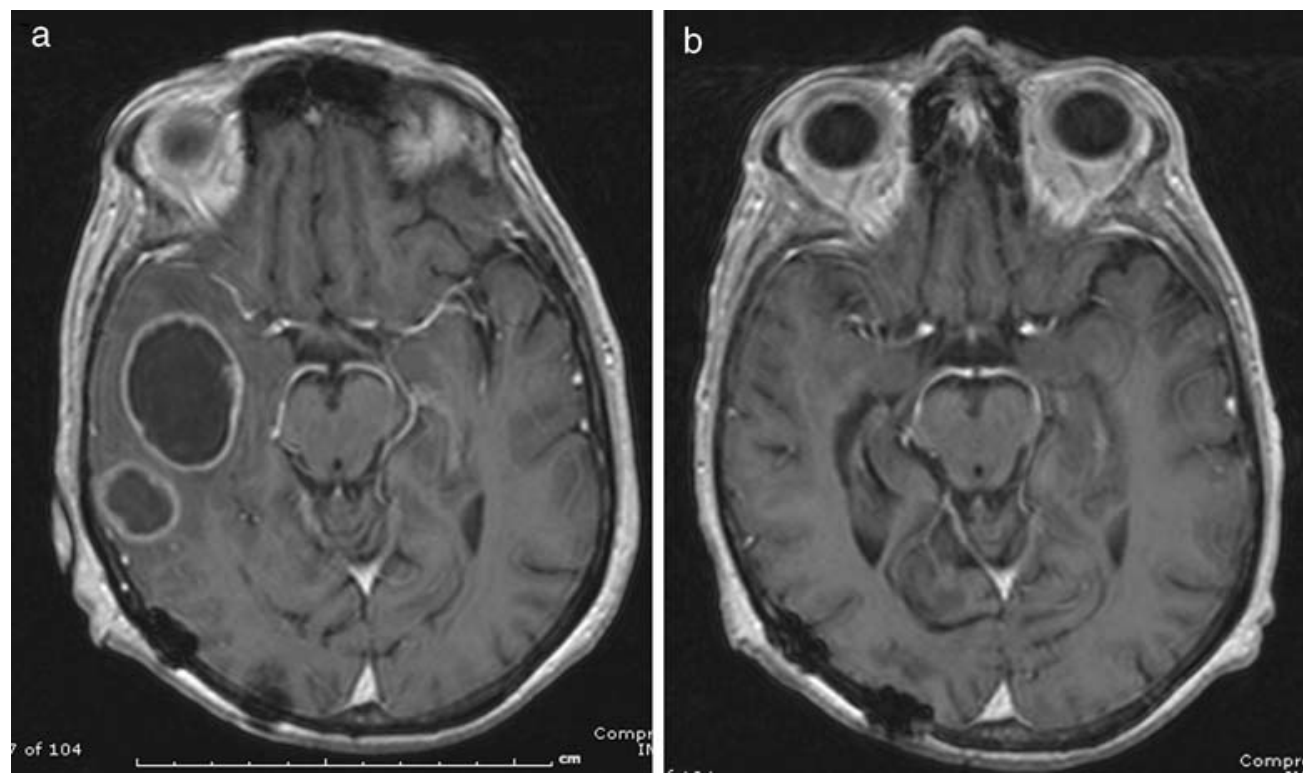

Fig. 9. A 69 year old woman was diagnosed with poorly differentiated ovarian adenocarcinoma in May 2001, at which time she underwent extensive abdominal and gynecologic surgery, followed by six cycles of taxol/ carboplatin. She presented a seizure in May 2002, and a metastatic lesion was identified in the right parietal region. She underwent a craniotomy for tumor resection, followed by eight cycles of the carboplatin regimen in conjunction with BBBD. She was considered in complete response (CR) after two cycles, and that condition was maintained until December 2005, when she relapsed in the right temporal lobe (a). BBBD treatments were resumed, and she was considered in CR after three cycles (b)

When reviewing the clinical results on $\mathrm{BBBD}$, all variables involved must be considered and adequately weighted against the surrogates under study.

In an attempt to decrease the number of variables involved, to nullify the choice of the chemotherapy agent and to specifically isolate the impact of delivery in the treatment of brain tumors, Kraemer et al. adopted an interesting approach (56). These authors studied the intensity of delivery against tumor response and survival in primary CNS lymphoma, a notoriously chemosensitive CNS tumor nonetheless depicting a poor outcome. Two surrogate measures of total dose intensity were used in the design of a delivery score: the number of disruption procedures over the treatment and a weighted quality of disruption score. In this study, the extent of the increase in permeability produced by the procedure correlated with long term survival in a population of patients treated

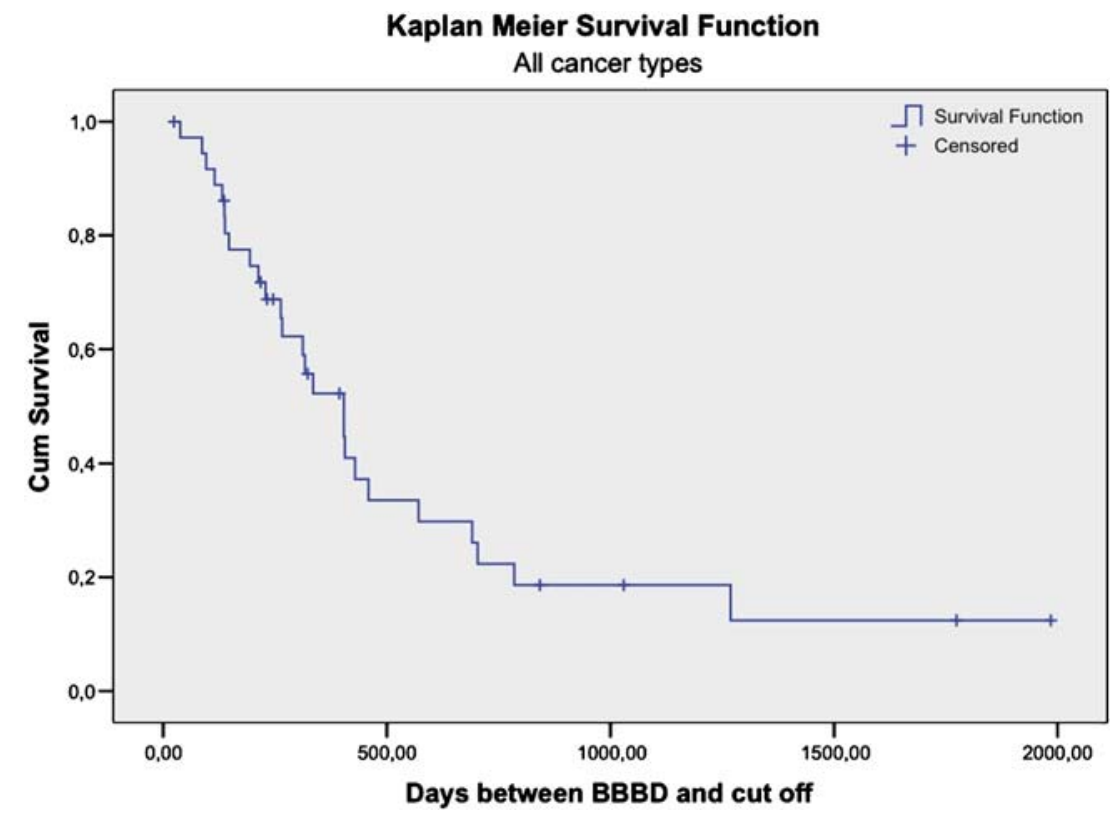

Fig. 10. Kaplan-Meier survival curve (days) for the global group of metastasis patients, from study entry to death 
with the BBBD procedure combined with intra-arterial methotrexate infusion, emphasizing the importance of delivery on the patient's outcome (49).

The procedure has been applied in the treatment of different brain tumor types. Williams et al. reported on the use of BBBD in conjunction with a regimen composed of intra-arterial carboplatin and intra-venous etoposide in 34 patients with various histologies (57). Of these 34 patients, 22 had measurable disease, and nine radiographic responses (50\% or more decrease in enhancing tumors) were observed in these patients. Carboplatin and etoposide delivered in conjunction to BBBD was considered an active regimen in the treatment of malignant astrocytomas and showed dramatic responses in primitive neuroectodermal tumors and CNS lymphoma. Additionally, the durability of responses in patients with disseminated CNS germ cell tumors is encouraging.

Kreamer et al. reported on the use of a similar protocol, using, in addition to the carboplatin and etoposide, a predisruption dose of i.v. cyclophosphamide in a series of 41 malignant astrocytoma patients (37). In this series, 28 patients were exposed to the BBBD treatment, whereas 13 patients received intra-arterial (IA) chemotherapy only. Although treatment modality (BBBD versus IA) scored as a highly significant variable both in uni- and multivariate analysis $(p=$ 0.0113), and thus BBBD patients survived significantly longer than IA patients, bias cannot be accounted for in this type of uncontrolled study. A median survival time of 90 weeks for the BBBD group was observed, compared to 50 weeks for the IA group.

The Centre Hospitalier Universitaire de Sherbrooke (CHUS) neuro-oncology group joined the consortium in 2000 and has recently reported results with the BBBD procedure combined with the administration of a carboplatinbased tri-drug regimen (58). We observed a prolonged median survival of 32.2 months in the treatment of glioma, more than double the reported median survivals of 9 to 14 months with standard therapeutic modalities (59-62). We also recently reported our clinical data on brain metastasis (53). We observed a spectacular improvement in median survival for brain metastasis from ovarian carcinoma, lymphomas and lung carcinoma. Our series depicted median survival of 24 months for ovarian carcinoma, 16 months for systemic lymphomas and 13 months for lung carcinoma, compared to reported median survival of 12 months, 3 months and 7 months respectively for ovarian, lymphoma and lung brain metastasis patient series (Figs. 9 and 10).

Recently, Hall et al. reported the experience of the consortium with diffuse pontine gliomas, a tumor type bearing a particularly poor prognosis, and typically affecting young patients (63). In this small series of eight patients exposed to BBBD and carboplatin or methotrexate, a median time to progression of 15 months, and a median survival of 27 months were deemed promising. In this type of tumor, survival beyond 2 years is exceptional, and it really is the first time that a chemotherapy trial is reported to impact outcome (64).

All in all, these results clearly hint at an improvement in brain tumor patient's outcome. Ultimately, the real question is whether or not the procedure can impact tumor response and patients survival. To ascertain this query thoroughly, randomized phase III studies would need to be conducted. As only a few centers are offering the BBBD procedure, and as these tumors are not prevalent diseases, these studies are particularly difficult to put together. However, recent results showing major improvement in the survival of patients affected by different types of brain tumors and treated via the BBBD approach should prompt major oncology organizations to consider osmotic BBBD as a viable therapeutic option, even though the logistic of the procedure is complex, and setup multi-center randomized trials.

\section{CONCLUSION}

The blood-brain barrier can no longer be ignored as a factor greatly limiting the entry of therapeutics in the CNS. According to Pardridge, over $98 \%$ of small molecules and close to $100 \%$ of large molecules do not cross the BBB (19). In neuro-oncology, clinical trials on brain tumor treatment are still designed with molecules that do not cross the BBB (e.g. vincristine, taxol). The peculiarities imposed by the $\mathrm{BBB}$ in the treatment of CNS diseases can no longer be ignored, and the delivery issue needs to be addressed appropriately. Less than $1 \%$ of pharmaceutical companies actually have a BBB drug targeting effort. The BBBD strategy initially proposed more than 3 decades ago has come a long way, venturing in the realm of clinical studies. However, its widespread acceptance has been hampered by many factors: the difficulty in setting teams dedicated to perform these procedures, the relative paucity of brain tumors, the difficulty of putting together randomized studies are but a few factors. However, concerted effort such as the international BBB consortium will allow the design of better multi-center trials eventually powered to answer whether or not this approach is worthwhile in the treatment of different CNS pathologies. As the clinical results obtained in the treatment of malignant gliomas and brain metastasis (representing the bulk of brain cancers) overall remains poor with traditional treatments, and very few major breakthroughs have been reported in the last decades despite active clinical research, clinical investigators tend to adopt a more open-minded approach toward alternative treatment strategies emphasizing CNS delivery. The promising results obtained in trials published on BBBD in the last decade will allow a better accrual of patients in clinical studies. However, promising results are just that, promising results, until randomized clinical studies can be put together and show a clear advantage of such an invasive technique on patient's survival. We are about to open such a randomized study in patients with oat cell carcinoma metastatic to the brain.

\section{ACKNOWLEDGEMENTS}

This work was supported by a grant from the Canadian Institute of Health Research (CIHR), and by the National Bank research chair on the treatment of brain tumors.

\section{REFERENCES}

1. D. Fortin. Altering the properties of the blood-brain barrier: disruption and permeabilization. Prog. Drug Res. 61:125-154 (2003). 
2. P. Ehrlich. Das sauerstoff-bedürfnis des organismus. Eine Farbenanalytische Studie, Hirschwald, Berlin, 1885.

3. P. Ehrlich. Ueber die beziehungen von chemischer constitution, vertheilung, und pharmakologischen wirkung. Collected Studies on Immunity, Wiley, Berlin, 1906, pp. 404-442.

4. E. Goldmann. Vitalfarbung am zentralnervensystem. Abhandl Konigl preuss Akad Wiss. 1:1-60 (1913).

5. W. M. Pardridge. Blood-brain barrier delivery of protein and non-viral gene therapeutics with molecular Trojan horses. $J$. Control Release 122(3):345-348 (2007), Oct 8.

6. L. L. Rubin, and J. M. Staddon. The cell biology of the bloodbrain barrier. Annu. Rev. Neurosci. 22:11-28 (1999).

7. H. Davson, and W. H. Oldendorf. Symposium on membrane transport. Transport in the central nervous system. Proc. R. Soc. Med. 4:326-329 (1967), Apr.

8. Z. Cohen, G. Bonvento, P. Lacombe, and E. Hamel. Serotonin in the regulation of brain microcirculation. Prog. Neurobiol. 50 (4):335-362 (1996), Nov.

9. Z. Cohen, G. Molinatti, and E. Hamel. Astroglial and vascular interactions of noradrenaline terminals in the rat cerebral cortex. J. Cereb. Blood Flow Metab. 17(8):894-904 (1997), Aug.

10. J. Fenstermacher, P. Gross, N. Sposito, V. Acuff, S. Pettersen, and K. Gruber. Structural and functional variations in capillary systems within the brain. Ann. N. Y. Acad. Sci. 529:21-30 (1988)

11. T. S. Reese, and M. J. Karnovsky. Fine structural localization of a blood-brain barrier to exogenous peroxidase. J. Cell Biol. 34 (1):207-217 (1967), Jul.

12. H. Wolburg, and A. Lippoldt. Tight junctions of the blood-brain barrier: development, composition and regulation. Vascul. Pharmacol. 38(6):323-337 (2002), Jun.

13. N. J. Abbott, L. Ronnback, and E. Hansson. Astrocyteendothelial interactions at the blood-brain barrier. Nat. Rev. Neurosci. 7(1):41-53 (2006), Jan.

14. J. F. Deeken, and W. Loscher. The blood-brain barrier and cancer: transporters, treatment, and Trojan horses. Clin. Cancer Res. 13(6):1663-1674 (2007), Mar 15.

15. M. W. Smith, and M. Gumbleton. Endocytosis at the blood-brain barrier: from basic understanding to drug delivery strategies. J. Drug Target. 14(4):191-214 (2006), May.

16. R. A. Kroll, and E. A. Neuwelt. Outwitting the blood-brain barrier for therapeutic purposes: osmotic opening and other means. Neurosurgery. 42(5):1083-1099 (1998), May; discussion 1099-1100.

17. W. M. Pardridge. The blood-brain barrier: bottleneck in brain drug development. NeuroRx. 2(1):3-14 (2005), Jan.

18. V. A. Levin. Relationship of octanol/water partition coefficient and molecular weight to rat brain capillary permeability. J. Med Chem. 23(6):682-684 (1980), Jun.

19. W. M. Pardridge. Blood-brain barrier delivery. Drug Discov. Today 12(1-2):54-61 (2007), Jan.

20. E. A. Neuwelt. Implications of the Blood-Brain Barrier and Its Manipulation. Vol 1 and 2, Plenum, New York, 1989.

21. E. M. Kemper, W. Boogerd, I. Thuis, J. H. Beijnen, and O. van Tellingen. Modulation of the blood-brain barrier in oncology: therapeutic opportunities for the treatment of brain tumours? Cancer Treat Rev. 30(5):415-423 (2004), Aug.

22. A. K. Ghose, V. N. Viswanadhan, and J. J. Wendoloski. A knowledge-based approach in designing combinatorial or medicinal chemistry libraries for drug discovery. 1. A qualitative and quantitative characterization of known drug databases. J. Comb. Chem. 1(1):55-68 (1999), Jan.

23. C. A. Lipinski. Drug-like properties and the causes of poor solubility and poor permeability. J. Pharmacol. Toxicol. Methods. 44(1):235-249 (2000), Jul-Aug.

24. R. L. Juliano, and V. Ling. A surface glycoprotein modulating drug permeability in Chinese hamster ovary cell mutants. Biochim. Biophys. Acta. 455(1):152-162 (1976), Nov 11.

25. C. Cordon-Cardo, J. P. O'Brien, J. Boccia, D. Casals, J. R. Bertino, and M. R. Melamed. Expression of the multidrug resistance gene product (P-glycoprotein) in human normal and tumor tissues. $J$. Histochem. Cytochem. 38(9):1277-1287 (1990), Sep.

26. S. I. Rapoport, M. Hori, and I. Klatzo. Testing of a hypothesis for osmotic opening of the blood-brain barrier. Am. J. Physiol. 223 (2):323-331 (1972), Aug.

27. W. M. Pardridge. Targeting neurotherapeutic agents through the blood-brain barrier. Arch. Neurol. 59(1):35-40 (2002), Jan.
28. M. W. B. Bradbury. Appraisal of the role of endothelial cells and glia in barrier breakdown. In A. Suckling, M. Rumsby, and M. Bradbury (eds.), The Blood-Brain Barrier in Health and Disease, Ellis Horwood, Chichester, 1986, pp. 128-129.

29. W. M. Pardridge. Advances in cell biology of blood-brain barrier transport. Semin. Cell Biol. 2(6):419-426 (1991), Dec.

30. W. Selman, W. Lust, and R. RA. Cerebral blood flow, McGrawHill, New York, 1996.

31. S. I. Rapoport. Effect of concentrated solutions on blood-brain barrier. Am. J. Physiol. 219(1):270-274 (1970), Jul.

32. M. W. Brightman, M. Hori, S. I. Rapoport, T. S. Reese, and E. Westergaard. Osmotic opening of tight junctions in cerebral endothelium. J. Comp. Neurol. 152(4):317-325 (1973), Dec 15.

33. K. Dorovini-Zis, P. D. Bowman, A. L. Betz, and G. W. Goldstein. Hyperosmotic arabinose solutions open the tight junctions between brain capillary endothelial cells in tissue culture. Brain Res. 302(2):383-386 (1984), Jun 8.

34. D. Fortin, C. Gendron, M. Boudrias, and M. P. Garant Enhanced chemotherapy delivery by intraarterial infusion and blood-brain barrier disruption in the treatment of cerebral metastasis. Cancer 109(4):751-760 (2007), Feb 15.

35. D. Fortin. [The blood-brain barrier should not be underestimated in neuro-oncology]. Rev. Neurol. (Paris). 160(5 Pt 1):523532 (2004), May.

36. D. Fortin, E. A. Neuwelt. Therapeutic manipulation of the blood-brain barrier. Neurobase-neurosurgery. 1st Ed. Medlink CD-ROM.

37. D. F. Kraemer, D. Fortin, and E. A. Neuwelt. Chemotherapeutic dose intensification for treatment of malignant brain tumors: recent developments and future directions. Curr. Neurol. Neurosci. Rep. 2(3):216-224 (2002), May.

38. R. Blasberg, D. Groothius, and P. Molnar. A review of hyperosmotic blood-brain barrier disruption in seven experimental brain tumor models. In B. B. Johansson, C. Owman, and H. Widner (eds.), Vol Pathophysiology of the Blood-Brain Barrier, Elsevier, Amsterdam, 1990, pp. 197-220.

39. B. Oztas, and M. Kucuk. Intracarotid hypothermic saline infusion: a new method for reversible blood-brain barrier disruption in anesthetized rats. Neurosci. Lett. 190(3):203-206 (1995), May 12.

40. R. A. Kroll, M. A. Pagel, L. L. Muldoon, S. Roman-Goldstein, S. A. Fiamengo, and E. A. Neuwelt. Improving drug delivery to intracerebral tumor and surrounding brain in a rodent model: a comparison of osmotic versus bradykinin modification of the blood-brain and/or blood-tumor barriers. Neurosurgery. 43 (4):879-886 (1998), Oct; discussion 886-879.

41. E. A. Neuwelt, P. A. Barnett, C. I. McCormick, L. G. Remsen, R. A Kroll, and G. Sexton. Differential permeability of a human brain tumor xenograft in the nude rat: impact of tumor size and method of administration on optimizing delivery of biologically diverse agents. Clin. Cancer Res. 4(6):1549-1555 (1998), Jun.

42. D. Fortin, R. Adams, and A. Gallez. A blood-brain barrier disruption model eliminating the hemodynamic effect of ketamine. Can. J. Neurol. Sci. 31(2):248-253 (2004), May.

43. L. G. Remsen, M. A. Pagel, C. I. McCormick, S. A. Fiamengo, G. Sexton, and E. A. Neuwelt. The influence of anesthetic choice, $\mathrm{PaCO} 2$, and other factors on osmotic blood-brain barrier disruption in rats with brain tumor xenografts. Anesth. Analg. 88 (3):559-567 (1999), Mar.

44. M. K. Gumerlock, and E. A. Neuwelt. The effects of anesthesia on osmotic blood-brain barrier disruption. Neurosurgery 26 (2):268-277 (1990), Feb.

45. D. Fortin, C. I. McCormick, L. G. Remsen, R. Nixon, and E. A. Neuwelt. Unexpected neurotoxicity of etoposide phosphate administered in combination with other chemotherapeutic agents after blood-brain barrier modification to enhance delivery, using propofol for general anesthesia, in a rat model. Neurosurgery 47 (1):199-207 (2000), Jul.

46. W. C. Cosolo, P. Martinello, W. J. Louis, and N. Christophidis. Blood-brain barrier disruption using mannitol: time course and electron microscopy studies. Am. J. Physiol. 256(2 Pt 2):R443447 (1989), Feb.

47. S. Roman-Goldstein, D. A. Clunie, J. Stevens, et al. Osmotic blood-brain barrier disruption: CT and radionuclide imaging. AJNR Am. J. Neuroradiol. 15(3):581-590 (1994), Mar. 
48. R. A. Rawson. The binding of T-1824 and structurally related diazo dyes by the plasma proteins. Am. J. Physiol. 138:708-717 (1942).

49. F. B. Freedman, and J. A. Johnson. Equilibrium and kinetic properties of the Evans blue-albumin system. Am. J. Physiol. 216(3):675-681 (1969), Mar.

50. A. W. Vorbrodt, D. H. Dobrogowska, M. Tarnawski, and A. S. Lossinsky. A quantitative immunocytochemical study of the osmotic opening of the blood-brain barrier to endogenous albumin. J. Neurocytol. 23(12):792-800 (1994), Dec.

51. N. D. Doolittle, M. E. Miner, W. A. Hall, et al. Safety and efficacy of a multicenter study using intraarterial chemotherapy in conjunction with osmotic opening of the blood-brain barrier for the treatment of patients with malignant brain tumors. Cancer 88(3):637-647 (2000), Feb 1.

52. D. Fortin, L. D. McAllister, G. Nesbit, et al. Unusual cervical spinal cord toxicity associated with intra-arterial carboplatin, intra-arterial or intravenous etoposide phosphate, and intravenous cyclophosphamide in conjunction with osmotic blood brain-barrier disruption in the vertebral artery. AJNR Am. J. Neuroradiol. 20(10):1794-1802 (1999), Nov-Dec.

53. S. C. Saris, R. G. Blasberg, R. E. Carson, et al. Intravascular streaming during carotid artery infusions. Demonstration in humans and reduction using diastole-phased pulsatile administration. J. Neurosurg. 74(5):763-772 (1991), May.

54. S. I. Rapoport. Modulation of blood-brain barrier permeability. J. Drug Target 3(6):417-425 (1996).

55. L. D. McAllister, N. D. Doolittle, P. E. Guastadisegni, et al. Cognitive outcomes and long-term follow-up results after enhanced chemotherapy delivery for primary central nervous system lymphoma. Neurosurgery 46(1):51-60 (2000), Jan; discussion 60-51.

56. D. F. Kraemer, D. Fortin, N. D. Doolittle, and E. A. Neuwelt. Association of total dose intensity of chemotherapy in primary central nervous system lymphoma (human non-acquired immuno- deficiency syndrome) and survival. Neurosurgery 48(5):1033-1040 (2001), May discussion 1040-1031.

57. P. C. Williams, W. D. Henner, S. Roman-Goldstein, et al. Toxicity and efficacy of carboplatin and etoposide in conjunction with disruption of the blood-brain tumor barrier in the treatment of intracranial neoplasms. Neurosurgery 37(1):17-27 (1995), Jul; discussion 27-18.

58. D. Fortin, A. Desjardins, A. Benko, T. Niyonsega, and M. Boudrias. Enhanced chemotherapy delivery by intraarterial infusion and blood-brain barrier disruption in malignant brain tumors: the Sherbrooke experience. Cancer 103(12):2606-2615 (2005), Jun 15.

59. M. Huncharek, and J. Muscat. Treatment of recurrent high grade astrocytoma; results of a systematic review of 1,415 patients. Anticancer Res. 18(2B):1303-1311 (1998), Mar-Apr.

60. M. Huncharek, J. Muscat, and J. F. Geschwind. Multi-drug versus single agent chemotherapy for high grade astrocytoma; results of a meta-analysis. Anticancer Res. 18(6B):4693-4697 (1998), Nov-Dec.

61. L. A. Stewart. Chemotherapy in adult high-grade glioma: a systematic review and meta-analysis of individual patient data from 12 randomised trials. Lancet. 359(9311):1011-1018 (2002), Mar 23.

62. R. Stupp, P. Y. Dietrich, S. Ostermann Kraljevic, et al. Promising survival for patients with newly diagnosed glioblastoma multiforme treated with concomitant radiation plus temozolomide followed by adjuvant temozolomide. J. Clin. Oncol. 20(5):13751382 (2002), Mar 1

63. W. A. Hall, N. D. Doolittle, M. Daman, et al. Osmotic bloodbrain barrier disruption chemotherapy for diffuse pontine gliomas. J. Neurooncol. 77(3):279-284 (2006), May.

64. J. L. Finlay, and S. Zacharoulis. The treatment of high grade gliomas and diffuse intrinsic pontine tumors of childhood and adolescence: a historical—and futuristic - perspective. J. Neurooncol. 75(3):253266 (2005), Dec. 